\title{
A randomised, placebo controlled, comparative trial of the gastrointestinal safety and efficacy of AZD3582 versus naproxen in osteoarthritis
}

\author{
L S Lohmander, D McKeith, O Svensson, M Malmenäs, L Bolin, A Kalla, G Genti, \\ J Szechinski, C Ramos-Remus, for the STAR Multinational Study Group
}

Ann Rheum Dis 2005;64:449-456. doi: 10.1136/ard.2004.023572

See end of article for authors' affiliations

Correspondence to: Professor Stefan

Lohmander, Department of Orthopaedics, Lund

University, Lund University

Hospital, SE-221 85 Lund,

Sweden;

Stefan.Lohmander@

ort.lu.se

Accepted 6 July 2004

Published Online First

2 September 2004

\begin{abstract}
Objective: To evaluate the gastrointestinal safety and efficacy of the COX inhibiting nitric oxide donator AZD3582 in patients with hip or knee osteoarthritis.

Methods: 970 patients were randomised (7:7:2) to AZD3582 $750 \mathrm{mg}$ twice daily, naproxen $500 \mathrm{mg}$ twice daily, or placebo twice daily in a double blind study. The primary end point was the six week incidence of endoscopic gastroduodenal ulcers (diameter $\geqslant 3 \mathrm{~mm}$ ). Overall damage measured on the Lanza scale was a secondary end point. Safety and tolerability assessments included endoscopic upper gastrointestinal erosions and the gastrointestinal symptom rating scale (GSRS). Efficacy was primarily assessed by WOMAC.

Results: The incidence of ulcers with AZD3582 was $9.7 \%$ and with naproxen $13.7 \%$ ( $p=0.07, N S), v 0 \%$ on placebo. The incidence of Lanza scores $>2$ was higher with naproxen (43.7\%) than with AZD3582 $(32.2 \%)(p<0.001)$. Compared with baseline, significantly fewer ulcers and erosions developed in stomach and stomach/duodenum combined, and fewer erosions developed in stomach, duodenum, and both combined on AZD3582 than on naproxen. GSRS reflux and abdominal pain subscale scores were lower for AZD3582 than for naproxen but there was no difference for indigestion, constipation, and diarrhoea. AZD3582 was as effective as naproxen at improving WOMAC scores. Both agents were well tolerated, with no significant effects on blood pressure.

Conclusions: At doses with similar efficacy in relieving osteoarthritis symptoms, the primary end point of six week endoscopic gastroduodenal ulcer incidence was not significantly different between AZD3582 and naproxen. Most secondary endoscopic gastrointestinal end points favoured AZD3582.
\end{abstract}

$\mathrm{N}$ on-steroidal anti-inflammatory drugs (NSAIDs) are effective in the treatment of acute and chronic pain in patients with osteoarthritis. ${ }^{12}$ Their anti-inflammatory and analgesic effects arise from the blockade of prostaglandin synthesis through inhibition of cyclo-oxygenase (COX) enzymes. However, this action results in well documented gastrotoxicity. ${ }^{3-6}$ In clinical endoscopy studies lasting from one to six months, the incidence of ulcers that are at least $3 \mathrm{~mm}$ in diameter in patients receiving non-selective NSAIDs (for example, naproxen, diclofenac, ibuprofen, piroxicam) ranges from $10 \%$ to $50 \% .^{7-13}$ Complications, such as perforation and bleeding, attributable to NSAID use occur in up to $1 \%$ of long term NSAID users, ${ }^{3}{ }^{14}$ with $15-35 \%{ }^{15}$ of the 15000 deaths a year in the USA and the 4000 deaths a year in England and Wales that involve peptic ulceration attributable to NSAID use. ${ }^{4} 1617$

The COX inhibiting nitric oxide donator (CINOD) class was developed for the treatment of acute and chronic pain. CINODs are designed to provide a multipathway mechanism of action of COX inhibition and controlled nitric oxide donation. In the gastrointestinal tract, nitric oxide mediates many processes that contribute to gastric mucosal integrity and, in particular, it exerts many of the same physiologically protective actions as prostaglandins. ${ }^{18}$ Thus it is hypothesised that donation of nitric oxide within the gastrointestinal tract may protect the mucosa from many of the adverse consequences of COX inhibition. ${ }^{18}{ }^{19}$ Representatives of this class are effective analgesic and anti-inflammatory agents with improved gastrointestinal safety profiles over nonselective NSAIDs in animal models. ${ }^{20}$
AZD3582 (4- ( nitro - oxy ) butyl - (2S ) - 2 - ( 6 - methoxy - 2 naphthyl)propanoate) was the first CINOD to be studied in large clinical trials. It donates nitric oxide both in vitro ${ }^{21}$ and in vivo (Adding LC et al, personal communication) and inhibits both COX-1 and COX-2. ${ }^{22}$ AZD3582 effectively and dose dependently reduced pain and inflammation in rat models, ${ }^{23}$ and was associated with less gastrointestinal injury than equimolar doses of naproxen in rat models of gastrointestinal safety. ${ }^{24-26}$ In a 12 day study of 31 healthy volunteers, ${ }^{27}$ there were significantly fewer gastroduodenal erosions on AZD3582 than on naproxen. Moreover, naproxen increased intestinal permeability, a marker of small intestinal damage, whereas AZD3582 and placebo did not. ${ }^{27}$ On the basis of these data, we evaluated the gastrointestinal safety, tolerability, and efficacy of AZD3582 in patients with osteoarthritis over a six week period. This is the first reported, randomised, controlled trial of a CINOD in patients with musculoskeletal disease.

\section{METHODS \\ Patients}

Men and women aged 40 to 75 years with symptomatic osteoarthritis of the knee or hip of at least three months' duration were recruited. All patients had radiographic

Abbreviations: ACR, American College of Rheumatology; COX, cyclooxygenase; CINOD, COX-inhibiting nitric oxide donator; GSRS, gastrointestinal symptom rating scale; ITT, intention to treat; NSAID, nonsteroidal anti-inflammatory drug; SF-36, 36 item short form health survey; VAS, visual analogue scale; WOMAC, Western Ontario and McMasters University osteoarthritis index 


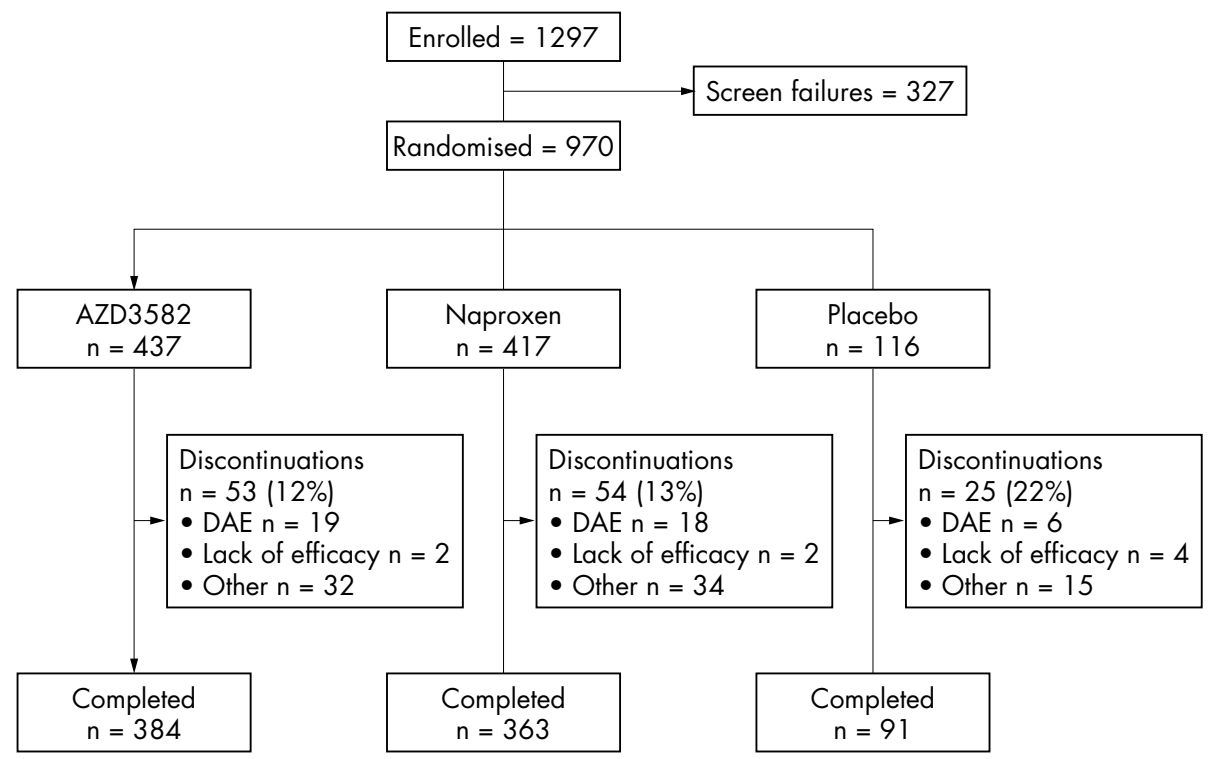

Figure 1 Patient disposition in a six week placebo controlled trial of the gastrointestinal safety and efficacy of AZD3582 and naproxen in patients with osteoarthritis.

evidence of hip or knee osteoarthritis (qualified as American College of Rheumatology (ACR) global functional class I, II, or III) and were current NSAID or paracetamol (acetaminophen) users. Helicobacter pylori status was assessed by serology at screening.

Patients were excluded from the study if they had any of the following: osteoarthritis secondary to inflammatory joint disease; a diagnosis of arthritis other than osteoarthritis; a history of gastric or duodenal bleeding within six months, or gastric or duodenal ulcer within three months; NSAID hypersensitivity; history of orthostatic hypotension; endoscopic ulcers at baseline screening. Patients on aspirin, $\mathrm{H}_{2}$ antagonists, antacids, misoprostol, proton pump inhibitors, or sucralfate were not eligible, and the use of these agents was prohibited throughout the study period.

\section{Study design}

The protocol was a six week, double blind, randomised, parallel group, placebo controlled study conducted at 80 sites: 15 in the United Kingdom, 12 in South Africa, eight in Poland, 13 in Hungary, 14 in Norway, six in Brazil, six in Argentina, and six in Mexico. The study was conducted from November 2001 to November 2002. It was carried out in accordance with the ethical principles in the Declaration of Helsinki, good clinical practice, and applicable regulatory requirements. The institutional review board or independent ethics committee of each participating centre provided ethical approval of the study protocol. All patients gave their written informed consent.

Subjects were randomised in a 7:7:2 ratio to AZD3582 $750 \mathrm{mg}$ twice daily, naproxen $500 \mathrm{mg}$ twice daily (which is

Table 1 Demographic and baseline characteristics

\begin{tabular}{|c|c|c|c|c|}
\hline \multirow[b]{2}{*}{ Variable } & \multicolumn{4}{|c|}{ Treatment group } \\
\hline & $\begin{array}{l}\text { AZD3582 } \\
750 \mathrm{mg} \\
(\mathrm{n}=437)\end{array}$ & $\begin{array}{l}\text { Naproxen } \\
500 \mathrm{mg} \\
(\mathrm{n}=417)\end{array}$ & $\begin{array}{l}\text { Placebo } \\
(n=116)\end{array}$ & $\begin{array}{l}\text { Total } \\
(n=970)\end{array}$ \\
\hline \multicolumn{5}{|l|}{ Demographic characteristics } \\
\hline Female sex & $316(72 \%)$ & $302(72 \%)$ & $88(76 \%)$ & $706(73 \%)$ \\
\hline Age (years) & $58.8(8.4)$ & $59.8(8.6)$ & $59.2(8.6)$ & $59.3(8.5)$ \\
\hline \multicolumn{5}{|l|}{ Race } \\
\hline White & $347(79 \%)$ & $335(80 \%)$ & $95(82 \%)$ & 777 (80\%) \\
\hline Black & $14(3 \%)$ & $14(3 \%)$ & $5(4 \%)$ & $33(3 \%)$ \\
\hline Other & $76(17 \%)$ & $68(16 \%)$ & $16(14 \%)$ & $160(16 \%)$ \\
\hline Primary study joint, knee (\%) (v hip) & $319(73 \%)$ & $290(69.6 \%)$ & $79(68.1 \%)$ & $688(70.9 \%)$ \\
\hline \multicolumn{5}{|l|}{ Baseline characteristics } \\
\hline Weight $(\mathrm{kg})$ & $81.3(16.4)$ & 79.5 (16.3) & $79.8(14.4)$ & $80.3(16.1)$ \\
\hline Height $(\mathrm{cm})$ & $164(9.4)$ & $163(10.0)$ & $163(8.7)$ & $164(9.6)$ \\
\hline Body mass index $\left(\mathrm{kg} / \mathrm{m}^{2}\right)$ & $30.3(5.6)$ & $29.8(5.2)$ & $30.1(5.7)$ & $30.0(5.5)$ \\
\hline Gastroduodenal erosions and ulcers* & $0.64(2.02)$ & $0.63(2.33)$ & $0.43(1.31)$ & $0.61(2.09)$ \\
\hline Gastroduodenal ulcers ( $\geqslant 3 \mathrm{~mm}$ diameter with depth) & $1(0.2 \%)$ & $2(0.5 \%)$ & $0(0.0 \%)$ & $3(0.3 \%)$ \\
\hline \multicolumn{5}{|l|}{ WOMAC score (mm) } \\
\hline Pain subscale & $47.2(20.5)$ & $45.1(21.0)$ & $45.0(20.1)$ & $46.0(20.7)$ \\
\hline Stiffness subscale & $51.3(22.9)$ & $49.0(24.0)$ & $49.9(25.0)$ & $50.1(23.6)$ \\
\hline Function subscale & $49.4(21.4)$ & $47.9(21.6)$ & $48.0(20.3)$ & $48.6(21.4)$ \\
\hline $\begin{array}{l}\text { Values are mean (SD) or } \mathrm{n}(\%) \text {. } \\
{ }^{*} 10 \text { erosions were considered to represent one ulcer. } \\
\text { WOMAC, Western Ontario and McMasters University }\end{array}$ & index. & & & \\
\hline
\end{tabular}




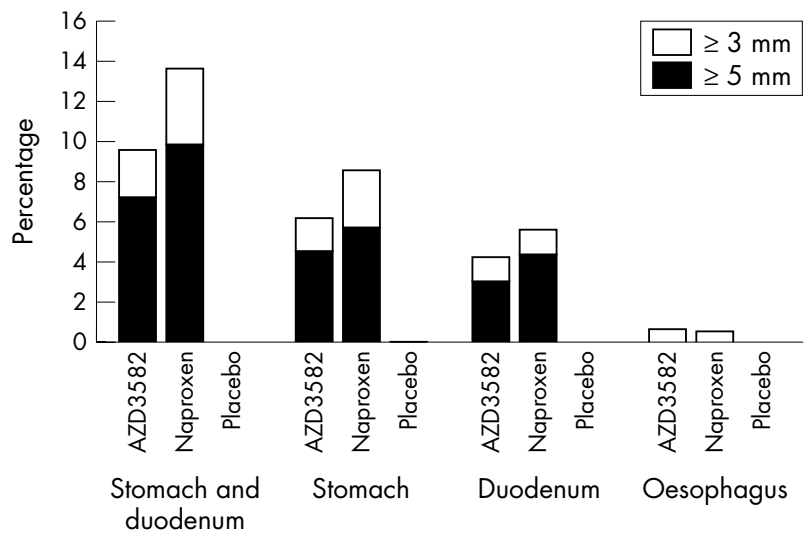

Figure 2 Incidence and location of ulcers $\geqslant 3 \mathrm{~mm}$ or $\geqslant 5 \mathrm{~mm}$ (with depth) in osteoarthritic patients treated with AZD3582 or naproxen.

an equimolar naproxen dose), or placebo twice daily. Study blinding was maintained by using capsules of AZD3582, naproxen, and placebo of identical appearance. Patients were instructed to take the treatment with food at 12 hour intervals. They were considered compliant to the treatment regimen if they took at least $70 \%$ of the study drug over the course of the study. Compliance was assessed by pill count.

Patients were assessed at an initial screening visit, and those who fulfilled the entry criteria discontinued NSAID treatment for two to 10 days before the baseline visit. Patients were allowed to take paracetamol up to $4000 \mathrm{mg}$ a day, provided by the investigator, for control of pain during the washout period. If the patient used paracetamol, it was requested that it be discontinued 12 hours before the baseline visit.

Safety, tolerability, and efficacy assessments were made at the screening and baseline visits, at weeks 1, 2, 3, 4, 5, and 6, and at the follow up visit (that is, within one week plus or minus three days of the end of the treatment).

\section{Gastrointestinal safety and tolerability assessments} Upper gastrointestinal endoscopy of the oesophagus, stomach, and duodenum was carried out according to local routines. Baseline endoscopy was conducted before the administration of the first dose of study drug. The second endoscopy was to be carried out within two days of the final dose. The primary end point of the study was the incidence of gastric or duodenal ulcers after six weeks of treatment. An ulcer was defined as a break in the mucosa of at least $3 \mathrm{~mm}$ in diameter with unequivocal depth, and was measured by close apposition of an endoscopic forceps with defined dimensions. In addition to number of ulcers, the size of the largest ulcer was recorded at each endoscopy.

To explore the properties of the ulcer size cut off employed, we undertook an ad hoc analysis using a minimum diameter of $5 \mathrm{~mm}$ as the criterion for an ulcer, instead of the $3 \mathrm{~mm}$ diameter that was specified as the primary variable.

Gastric damage was also scored using the Lanza scale. ${ }^{28}$ The proportion of patients with more than two erosions or an ulcer (Lanza score $>2$ ) was a secondary end point. Other secondary end points for gastrointestinal safety were the within-subject change between baseline and end of treatment in the number of oesophageal, gastric, and duodenal ulcers, erosions, and petechiae.

Gastrointestinal tolerability was assessed using the gastrointestinal symptom rating scale (GSRS). ${ }^{29}$ The GSRS contains 15 questions covering five major gastrointestinal symptoms: abdominal pain, reflux, indigestion, diarrhoea, and constipation. Patients were asked to rate discomfort from each gastrointestinal symptom on a seven point Likert scale, where 1 represents "no discomfort" and 7 represents "very severe discomfort." The GSRS was applied at the screening and baseline visits as well as on visits at weeks 1, 2, 4, and 6 .

\section{Efficacy assessments}

The Western Ontario and McMaster Universities (WOMAC) osteoarthritis index, version 3.1 visual analogue scale (VAS), ${ }^{30}$ and the 36 item short form health survey (SF-36) ${ }^{31}$ were used to assess response to treatment at baseline, at weeks 1, 2, 4, and 6, and at the follow up visit. Patients answered the WOMAC subscales of pain, stiffness, and physical function using a 48 hour recall period. The acute version of SF-36, with a recall period of one week, was used to evaluate the physical and mental wellbeing of the patients.

Efficacy was also assessed at the six week visit by the subjects' and physicians' overall rating of treatment, which were five point Likert scales (where 1 represents "very poor" and 5 represents "very good") by answering the following questions:

- Patient: "How do you rate your treatment overall, taking both pain relief and everything else into consideration?"

Table 2 Secondary endoscopic end point results (Lanza and erosion scores) at week 6

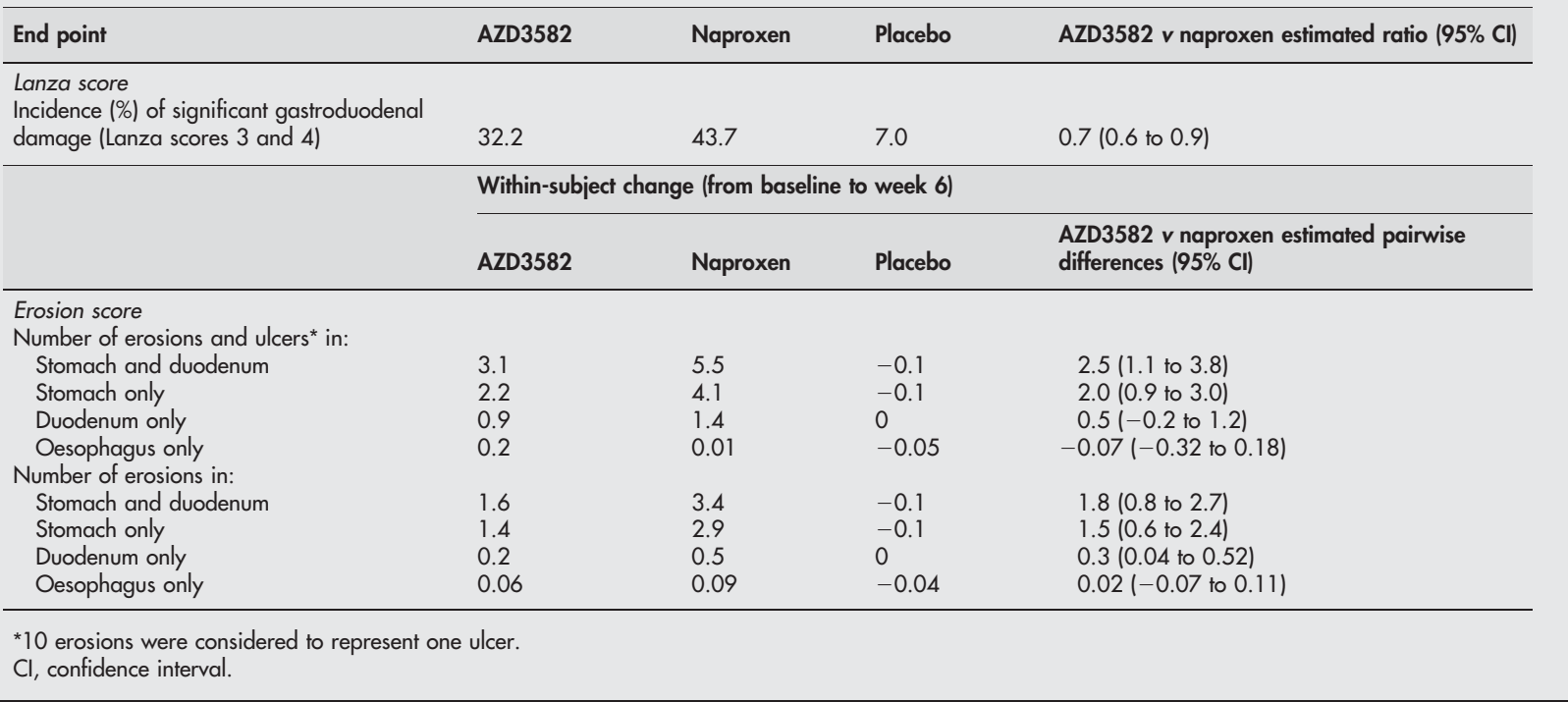


Table 3 Mean change in WOMAC subscales from baseline for knee and hip combined, and for knee and hip separately

\begin{tabular}{|c|c|c|c|c|}
\hline \multirow[b]{2}{*}{ WOMAC subscale } & \multicolumn{4}{|c|}{ Mean within-subject change from baseline to mean of Weeks 4 and 6} \\
\hline & AZD3582 & Naproxen & Placebo & $\begin{array}{l}\text { AZD3582 v naproxen estimated } \\
\text { pairwise differences }(95 \% \mathrm{CI})\end{array}$ \\
\hline $\begin{array}{l}\text { Knee and hip } \\
\text { Pain } \\
\text { Stiffness } \\
\text { Function }\end{array}$ & $\begin{array}{l}(n=424) \\
-15.9 \\
-18.0 \\
-15.3\end{array}$ & $\begin{array}{l}(n=405) \\
-14.7 \\
-16.9 \\
-14.9\end{array}$ & $\begin{array}{l}(n=109) \\
-5.8 \\
-8.6 \\
-6.1\end{array}$ & $\begin{array}{l}0.54(-1.87 \text { to } 2.95) \\
0.23(-2.45 \text { to } 2.91) \\
0.17(-2.11 \text { to } 2.45)\end{array}$ \\
\hline $\begin{array}{l}\text { Knee } \\
\text { Pain } \\
\text { Stiffness } \\
\text { Function }\end{array}$ & $\begin{array}{l}(n=308) \\
-17.0 \\
-19.7 \\
-16.8\end{array}$ & $\begin{array}{l}(n=281) \\
-16.0 \\
-19.1 \\
-16.3\end{array}$ & $\begin{array}{l}(n=76) \\
-7.0 \\
-9.4 \\
-7.3\end{array}$ & $\begin{array}{r}0.04(-2.78 \text { to } 2.86) \\
-0.61(-3.77 \text { to } 2.55) \\
0.04(-2.76 \text { to } 2.67)\end{array}$ \\
\hline $\begin{array}{l}\text { Hip } \\
\text { Pain } \\
\text { Stiffness } \\
\text { Function }\end{array}$ & $\begin{array}{l}(n=116) \\
-12.9 \\
-13.3 \\
-11.1\end{array}$ & $\begin{array}{l}(n=124) \\
-11.9 \\
-12.0 \\
-11.7\end{array}$ & $\begin{array}{l}(n=33) \\
-3.0 \\
-6.7 \\
-3.2\end{array}$ & $\begin{array}{r}1.30(-3.31 \text { to } 5.91) \\
1.49(-3.52 \text { to } 6.51) \\
-0.07(-4.22 \text { to } 4.09)\end{array}$ \\
\hline
\end{tabular}

- Investigator: "How do you rate the subject's treatment overall, taking both pain relief and everything else into consideration"

\section{General safety}

Patients were questioned by the investigator at each visit about the occurrence of any adverse event. For all adverse events, the investigator recorded the intensity, the seriousness, the action taken, and the outcome. Other assessments included haematology, clinical chemistry, urine analysis, pulse, blood pressure, and electrocardiogram. The blood pressure was measured with a calibrated manometer supine after 15 minutes of rest, and after two and five minutes of standing. At the request of an Independent Safety Monitoring Board (ISMB), the initial 401 patients enrolled in the study had additional assessments of pulse and blood pressure at one, two, and three hours after the first dose, and two to three hours after dosing following three days and one week of treatment.

\section{Statistical analysis}

The primary objective was to compare the proportion of patients with gastroduodenal ulcers after six weeks' treatment with AZD3582 or naproxen, using a two sided 95\% confidence interval (CI) for the AZD3582/naproxen ratios of proportions. The confidence interval was based on a generalised linear model with log as link function and a binomial distribution taking country into account. Studying 350 patients in each of the two groups made it possible to show a $50 \%$ reduction with a power of at least $80 \%$ with AZD 3582 , assuming $4-6 \%$ of patients would get ulcers on AZD3582 and $20-25 \%$ on naproxen.

The within-subject differences between baseline and the mean of treatment weeks 4 and 6 were used to analyse changes in WOMAC subscale scores and GSRS subscales. For the SF-36, the within-subject differences between baseline and the end of treatment for each subscale were analysed. An analysis of covariance (ANCOVA) approach with adjustment for country, baseline, and treatment was used for the pairwise comparisons between the different treatment groups when analysing WOMAC, GSRS, and SF-36. No correction for multiple comparisons was to be made.

Efficacy and safety analyses were carried out on all randomised patients who received at least one dose of the study preparation. The analysis of the primary end point involved an initial pairwise comparison of the ratio of the incidence of gastroduodenal ulcers with naproxen and placebo. The statistical analysis only allowed a comparison of AZD3582 and naproxen if a significant difference had been detected between naproxen and placebo.

A supplementary per-protocol analysis was carried out. This included all patients taking at least $70 \%$ of the investigational product, having the second endoscopy within two days before or after the last intake of the study drug, and without any other major protocol deviations judged to have an impact on the endoscopy result.

\section{RESULTS \\ Patients}

In all, 1297 patients were enrolled in the study, of whom 970 were randomised and received at least one dose of investigational product; 838 completed the six week long trial (fig 1). There were 898 patients ( 100 in the placebo group, 404 in the AZD3582 group, and 394 in the naproxen group) who had two endoscopies within or outside the protocol-specified two days before or after the last intake of study drug, and were included in the analysis of the endoscopy based variables (ITT). In the supplementary per-protocol analysis, 686 subjects were included (77 in the placebo group, 314 in the AZD3582 group, and 295 in the naproxen group). Of the 212 subjects excluded, 184 exclusions were because the second endoscopy was outside the specified two days. These exclusions were well balanced across the three groups. The remaining subjects were excluded because they received a prohibited drug (1.6\% of the AZD3582 group, $0.7 \%$ of the naproxen group, $3.4 \%$ of the placebo group), took less than

Table 4 Rating of response to treatment

\begin{tabular}{|c|c|c|c|c|}
\hline & \multirow{2}{*}{$\begin{array}{l}\text { Overall rating of treatment } \\
\text { (AZD3582 v naproxen pairwise } \\
\text { comparison (p)) }\end{array}$} & \multicolumn{3}{|c|}{ Number $(\%)$ rating response to treatment as "good" or "very good" } \\
\hline & & AZD3582 & Naproxen & Placebo \\
\hline Subjects & 0.50 & 309 (73.2) & $294(72.3)$ & $50(45.4)$ \\
\hline Investigators & 0.87 & $299(70.7)$ & $285(70.2)$ & 49 (44.6) \\
\hline
\end{tabular}




\begin{tabular}{|c|c|c|c|}
\hline Events & $\begin{array}{l}\text { AZD3582 } \\
(n=437)\end{array}$ & $\begin{array}{l}\text { Naproxen } \\
(\mathrm{n}=417)\end{array}$ & $\begin{array}{l}\text { Placebo } \\
(n=116)\end{array}$ \\
\hline \multicolumn{4}{|l|}{ Number of patients (\%): } \\
\hline With any adverse event & $310(71)$ & $295(71)$ & $77(66)$ \\
\hline With any serious adverse event ${ }^{\star}$ & $3(1)$ & $2(0)$ & $1(1)$ \\
\hline Discontinued because of adverse event & $16(4)$ & $16(4)$ & $6(5)$ \\
\hline \multicolumn{4}{|l|}{ Adverse event $(\%) \dagger$} \\
\hline Headache & $166(38)$ & $152(36)$ & $42(36)$ \\
\hline Back pain & $31(7)$ & $38(9)$ & $11(9)$ \\
\hline Arthralgia & $24(5)$ & $38(9)$ & $13(11)$ \\
\hline Nasopharyngitis & $25(6)$ & $24(6)$ & $6(5)$ \\
\hline Pain in limb & $22(5)$ & $19(5)$ & $6(5)$ \\
\hline Influenza & $17(4)$ & $7(2)$ & $4(3)$ \\
\hline Dizziness & $8(2)$ & $12(3)$ & $5(4)$ \\
\hline Pharyngitis & $15(3)$ & $10(2)$ & $4(3)$ \\
\hline \multicolumn{4}{|l|}{ NSAID related GI symptoms (\%) } \\
\hline Dyspepsia & $37(8)$ & $41(10)$ & $8(7)$ \\
\hline Upper abdominal pain & $25(6)$ & $43(10)$ & $10(9)$ \\
\hline Nausea & $28(6)$ & $21(5)$ & $6(5)$ \\
\hline Abdominal pain & $18(4)$ & $25(6)$ & $3(3)$ \\
\hline Diarrhoea & $23(5)$ & $18(4)$ & $4(3)$ \\
\hline Constipation & $17(4)$ & $19(5)$ & $2(2)$ \\
\hline Abdominal distension & $18(4)$ & $8(2)$ & $4(3)$ \\
\hline Flatulence & $15(3)$ & $11(3)$ & $3(3)$ \\
\hline Total incidence & $124(28)$ & $132(32)$ & $32(28)$ \\
\hline \multicolumn{4}{|c|}{$\begin{array}{l}\text { *Four in the AZD } 3582 \text { group (otorrhoea (day 49), breast cancer (day }-2 \text { ), duodenal ulcer and gastric erosions } \\
\text { (day 2)), two in the naproxen group (diabetes mellitus (day 42) and unstable angina (day 49)); one in the placebo } \\
\text { group (intestinal diverticulum (day 27)). } \\
\text { tlncidence } \geqslant 3 \% \text { in any treatment group. } \\
\text { GI, gastrointestinal; NSAID, non-steroidal anti-inflammatory drug. }\end{array}$} \\
\hline
\end{tabular}

$70 \%$ of investigational product $(3.4 \%$ AZD 3582, $3.4 \%$ naproxen, $5.2 \%$ placebo), or had other important deviations from the protocol $(2.1 \%$ AZD 3582, $2.4 \%$ naproxen, $1.7 \%$ placebo).

The treatment groups were well balanced at baseline for demographic characteristics, joints affected by osteoarthritis, $H$ pylori status, and ACR functional class (table 1 ). There were no differences in baseline endoscopy scores between treatment groups. Further, there were no differences in baseline WOMAC scores between treatment groups or between those with hip or knee osteoarthritis. The baseline scores for all SF36 domains were similar between treatment groups, as were the proportions of subjects taking rescue medication for osteoarthritis related pain (data not shown). Three subjects with ulcer at baseline were erroneously included in the trial (table 1).

\section{Gastroduodenal ulcer}

None of the patients on placebo had gastroduodenal ulcers by six weeks of treatment, compared with $13.7 \%$ in the naproxen group and $9.7 \%$ in the AZD3582 group. Compared with placebo, the incidence of gastroduodenal ulcers was higher with both AZD3582 $(\mathrm{p}<0.002)$ and naproxen $(\mathrm{p}<0.0001)$. The ratio in ulcer incidence between AZD3582 and naproxen was 0.70 (95\% CI, 0.48 to 1.03 ) and did not achieve statistical significance $(p=0.07)$. The results of the per-protocol analysis were similar $(10.2 \%$ ulcers with AZD3582 $v 12.5 \%$ with naproxen, $\mathrm{p}=0.32$ ). The relative effects of the two treatments were similar in patients who were $H$ pylori negative or positive at baseline (data not shown) and for ulcers $\geqslant 5 \mathrm{~mm}$ in size $(7.2 \%$ on AZD3582, $9.9 \%$ on naproxen, $\mathrm{p}=0.18$, fig 2 ).

\section{Secondary end points}

Table 2 shows the nine prespecified secondary endoscopic end points. AZD3582 was associated with significantly less mucosal injury than naproxen when scored on the Lanza scale, or in terms of the number of erosions and ulcers in the stomach and duodenum combined or stomach alone, and the number of erosions in stomach and duodenum combined, stomach alone, or duodenum alone. There were few duodenal ulcers and little oesophageal injury, so that differences in the number of erosions and ulcers in the duodenum alone and injury scores for the oesophagus were not significantly different between the two active treatments.

\section{Symptoms and quality of life}

On the GSRS scale, treatment with AZD3582 was associated with an improved within-subject difference between baseline and treatment in weeks $4-6$ of 0.14 (95\% CI, 0.03 to 0.25 ) $(p=0.011)$ for reflux and $0.11(0.02$ to 0.2$)(p=0.016)$ for abdominal pain, compared with naproxen. There were no significant differences between AZD3582 and naproxen in the GSRS indigestion, constipation, and diarrhoea subscales,

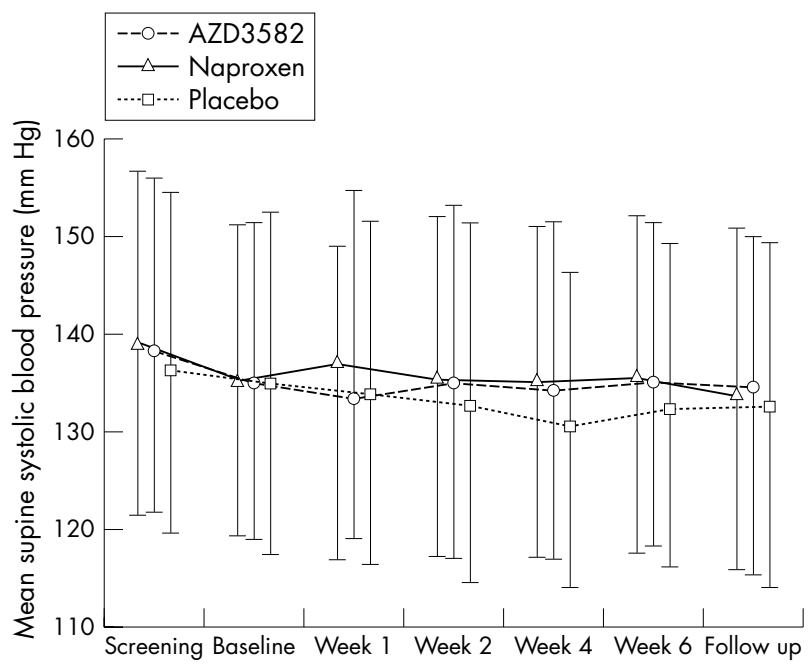

Figure 3 Mean supine systolic blood pressure in patients with osteoarthritis receiving AZD3582, naproxen, or placebo. Error bars = SD. 
neither were there significant differences in changes from baseline in any of the GSRS subscales for the pairwise comparison of placebo with AZD3582 or naproxen. AZD3582 and naproxen provided significant improvements over placebo in six of eight SF-36 domains: bodily pain, physical functioning, role-physical, vitality, mental health, and social functioning.

\section{Efficacy}

As shown in tables 3 and 4, AZD3582 and naproxen were of similar efficacy and significantly better than placebo. The WOMAC subscales showed this to be true for both knee and hip combined, and for knee and hip separately, although the efficacy at the knee was somewhat better than for the hip (table 3 ). The two active treatments offered greater improvements than placebo in the subjects' and physicians' overall rating of treatment $(\mathrm{p}<0.0001$ for both comparisons $)$, and there were no significant differences between AZD3582 and naproxen for either overall rating (table 4). Use of rescue medication (paracetamol) was similarly decreased by AZD3582 or naproxen compared with placebo (data not shown).

\section{Adverse events}

Adverse events occurred in 310 patients $(71 \%)$ in the AZD3582 group, $295(71 \%)$ in the naproxen group, and 77 $(66 \%)$ in the placebo group. There were similar incidences and types of clinical adverse events in the three treatment groups (table 5). In particular, the incidence of any gastrointestinal NSAID related adverse events-such as nausea, abdominal pain, or dyspepsia-was similar between groups, with incidences of $28 \%$ for AZD3582, 32\% for naproxen, and $28 \%$ for placebo. Most adverse events were mild to moderate in severity. There were seven serious adverse events reported in six subjects. Similar proportions of patients in each treatment group withdrew because of adverse effects (table 5 ).

\section{Blood pressure}

Overall, there were no significant differences between the treatment groups in terms of mean supine systolic (fig 3), diastolic, or mean orthostatic blood pressure (data not shown). The percentage of patients with a systolic blood pressure decrease from baseline of more than $30 \mathrm{~mm} \mathrm{Hg}$ did not differ between the treatment groups. More subjects on AZD3582 (19\%) and naproxen (16\%) had a decrease in diastolic blood pressure of greater than $15 \mathrm{~mm} \mathrm{Hg}$ than in the placebo group (10\%), but the proportions with a decrease of $30 \mathrm{~mm} \mathrm{Hg}$ or more did not differ. These changes were generally well tolerated and did not usually give rise to any symptoms. However, four subjects on AZD3582 had hypotension or decreased blood pressure recorded as an adverse event (versus none on naproxen or placebo), although this did not lead to withdrawal from the study. There were three protocol discontinuations because of a supine systolic blood pressure of $100 \mathrm{~mm} \mathrm{Hg}$ or less (two in the AZD3582 and one in the naproxen group). In the subset of 401 subjects in whom extra assessments were done at one, two, and three hours after first intake of drugs or placebo, there was a decrease in mean systolic and diastolic blood pressure of 6-8 $\mathrm{mm} \mathrm{Hg}$ with AZD3582. The effect was less after three to five days, and had diminished further after five to nine days.

\section{DISCUSSION}

AZD3582 donates nitric oxide both in vitro ${ }^{32}$ and in vivo (Adding LC et al, personal communication). This has been hypothesised to protect the gastrointestinal mucosa from many of the adverse consequences of COX inhibition by preserving adequate blood flow and by increasing protective mucus and bicarbonate secretion. ${ }^{18} 19$ The protective effect that was shown earlier against erosions in healthy volunteers ${ }^{27}$ could not be found against ulcers in patients with osteoarthritis in the present study. The incidence of gastroduodenal ulcers for AZD3582 was not significantly less than for naproxen, and both were higher than for placebo.

It is difficult to compare the ulcer incidence of $13.7 \%$ for naproxen in the present study with other studies, as the design, duration, and patient population differ across studies. Many studies are 12 weeks long, involve more than two endoscopies, and also involve patients with rheumatoid arthritis. The ulcer incidence from naproxen in studies involving osteoarthritic patients has varied between $10 \%$ and $19 \%,{ }^{33} 34$ but has been reported to be up to $26 \%$ for patients with rheumatoid arthritis. ${ }^{8}$ The gastroduodenal ulceration rates for COX-2 selective NSAIDs in patients with osteoarthritis were reported to be about 3\% after six weeks $^{12}{ }^{13}$ and between $3 \%$ and 5\% after 12 weeks. ${ }^{9}$

The sample size of 350 patients in each active group was chosen assuming true underlying proportions of 4-6\% ulcers for AZD3582 and 20-25\% for naproxen. At the end of the trial, 404 patients in the AZD3582 group and 394 in the naproxen group were analysed. With an ulcer incidence of $14 \%$ for naproxen it would then still be possible to achieve a significant difference with at least $80 \%$ power if the ulcer incidence for AZD3582 were half that of naproxen-that is, around $7 \%$.

Most six week studies have shown ulcer incidences of $3-4 \%$ for placebo, ${ }^{8}{ }^{913}$ so the placebo gastroduodenal ulcer rate of $0 \%$ in the present study is low but not unique. Hawkey et $a{ }^{12}$ in a 24 week long study where the six week data were presented separately, ${ }^{35}$ showed no ulcers in 182 patients on placebo. One reason for the low figure in the present study could be that patients with a history of recent upper gastrointestinal events were excluded. This was done for ethical reasons, as these patients are at high risk of developing new and potentially life threatening ulcer complications, but it should also add to the validity and clinical relevance of the study. Further, patients with one or more ulcers at the start of the study were excluded to allow a more easy evaluation of the influence of the study preparation on endoscopic findings. It was also considered more ethical, as those with significant lesions at inclusion would have had a higher risk of developing complications.

In previous studies, it has been common practice to include study patients having their gastroscopy up to seven days after last intake of investigational product in gastroduodenal endoscopic studies. ${ }^{12}{ }^{13}$ In the present study, the second gastroscopy was to be done no later than two days after the last intake of investigational product, and this was achieved in $84 \%$ of patients, with $97 \%$ no later than seven days. That the results of the per-protocol analysis were similar to the intent to treat population gives further support to the robustness of the study results.

The clinical significance of erosions is under debate. In a recent study it was found that patients with a baseline gastric ulcer had twice as many gastric erosions as those without ulcer (Yeomans ND, personal communication), and gastroduodenal erosions at baseline have been shown to increase the risk for endoscopically detected ulcers and clinical bleeds. ${ }^{36}$ The Lanza scale is a practical tool that combines erosions and ulcers and has been used in many studies. ${ }^{28} 3738$ Although there was a significant difference between AZD3582 and naproxen for the Lanza scale for erosions alone both in the stomach and duodenum and for erosions and ulcers combined in the stomach only, these results did not translate into a statistically significant difference for the primary end point. 
AZD3582 and naproxen were generally well tolerated, with similar adverse event rates. It is unclear whether the small but statistically significant differences between AZD3582 and naproxen in the two GSRS dimensions of reflux and abdominal pain are clinically relevant. Changes of 0.5 in the GSRS scale have been considered to be clinically significant in trials of proton pump inhibitors, but these changes represent decreases in the score from a different baseline value. ${ }^{39}$ For example, in a study of omeprazole, patients had mild discomfort at baseline (that is, 3 on the Likert scale) which decreased towards minor discomfort ( 2 on the Likert scale), ${ }^{40}$ whereas patients in the present study started with no discomfort ( 1 on the scale), but discomfort increased.

The decrease in blood pressure, possibly related to nitric oxide donation, which was initially apparent in the patients having extra blood pressure assessments, had diminished after one week. No further differences between treatment groups at subsequent visits were noted. This may have been related to the subsequent lack of specified timing of blood pressure measurements with respect to intake of investigational product; alternatively, nitrate tolerance may have occurred. The blood pressure lowering effects were generally well tolerated and subjects were typically asymptomatic.

In conclusion, AZD3582 had similar analgesic efficacy to naproxen. The $30 \%$ difference in the incidence of gastroduodenal ulcers after six weeks of treatment between AZD3582 and naproxen was not statistically significant.

\section{ACKNOWLEDGEMENTS}

We thank Professor C J Hawkey, Wolfson Digestive Diseases Centre, University Hospital Nottingham, for valuable discussions on the results and for review of the manuscript. The study was supported by a grant from AstraZeneca R\&D Södertälje.

\section{Authors' affiliations}

L S Lohmander, Department of Orthopaedics, Medical Faculty, Lund University, Lund, Sweden

D McKeith, Castlemilk Group Practice, Castlemilk Health Centre, Glasgow, UK

O Svensson, M Malmenäs, L Bolin, AstraZeneca R\&D, Södertälje, Sweden

A Kalla, Groote Schuur Hospital, Cape Town, South Africa

G Genti, Department of Rheumatology and Physiotherapy, Flor Ferenc Kórház, Rheumatológia, Kistarcsa, Hungary

J Szechinski, Department of Rheumatology, Medical University of Wroclaw, Wroclaw, Poland

C Ramos-Remus, Department of Rheumatology, Unidad de Investigacion en Enfermedades Cronico Degenerativas SC, Guadalajara, Jalisco, Mexico

The members of the STAR Multinational Study Group are listed in the appendix

\section{APPENDIX}

\section{The STAR Multinational Study Group}

Argentina: L Catoggio, E Gutfraind, J Duhau, E Mysler, J Marcos, O Messina. Brazil: M L Cardoso Pucielli, J Batista de Miranda, M Barros Bértolo, C Goldenstein Schainberg, J F Marques Neto, N R Severino. Hungary: F Gyulai, E Koó, F Szanyó, A Insperger, L Náfrádi, T Balázs, A Búvár, A Molnár, E Gömöri, J Náfrádi, Z Szekanecz, K Tarján, A Major. Mexico: R Borbon, R A Figueroa Gama, F Irazoque, L J Jara, L Lino Perez. Norway: T Forthun, S A Lonning, O Vassel, H Hövik, K Mariadasan, A Vedvik, H Fonnelöp, A Tandberg, Å N Hansen, T Tomala, B Jordet, B O Bache, A Kubak, O E Bo, R Stene, $\mathrm{T}$ M Pedersen, M Andersen, J F Nilsen. Poland: A Wall, J Lacki, S Mackiewicz, M Glowacka, E Kucharz, K Suchon, J Blacha, K Kwiatkowski. South Africa: S Lipschitz, M Tikly, E M van Duuren, N David, J Terblanche, C Lyddell, I Louw,
G Mody, I Anderson, H Nell, M V Middle. United Kingdom: C McKinnon, H P McGoldrick, M Salman, C Harding, S Taylor, J Robinson, J Maroni, J Fraser, S Sharma, J James, C Horne, M Anderson, B Norton, B Glekin.

\section{REFERENCES}

1 ACR. Recommendations for the medical management of osteoarthritis of the hip and knee: 2000 update. American College of Rheumatology subcommittee on osteoarthritis guidelines. Arthritis Rheum 2000;43:1905-15.

2 Pendleton A, Arden N, Dougados M, Doherty M, Bannwarth B, Bijlsma JW, et al. EULAR recommendations for the management of knee osteoarthritis: report of a task force of the standing committee for international clinical studies including therapeutic trials (ESCISIT). Ann Rheum Dis 2000;59:936-44.

3 Laine L. Approaches to nonsteroidal anti-inflammatory drug use in the highrisk patient. Gastroenterology 2001;120:594-606.

4 Singh G, Triadafilopoulos G. Epidemiology of NSAID induced gastrointestinal complications. J Rheumatol 1999;26:18-24.

5 Fries JF, Miller SR, Spitz PW, Williams CA, Hubert HB, Bloch DA. Toward an epidemiology of gastropathy associated with nonsteroidal antiinflammatory drug use. Gastroenterology 1989;96:647-55.

6 Wallace JL, McKnight W, Reuter BK, Vergnolle N. NSAID-induced gastric damage in rats: requirement for inhibition of both cyclooxygenase 1 and 2 . Gastroenterology 2000;119:706-14.

7 Agrawal NM, Caldwell J, Kivitz AJ, Weaver AL, Bocanegra TS, Ball J, et al. Comparison of the upper gastrointestinal safety of Arthrotec 75 and nabumetone in osteoarthritis patients at high risk for developing nonsteroidal anti-inflammatory drug-induced gastrointestinal ulcers. Clin Ther 1999;21:659-74

8 Simon LS, Weaver AL, Graham DY, Kivitz AJ, Lipsky PE, Hubbard RC, et al. Anti-inflammatory and upper gastrointestinal effects of celecoxib in rheumatoid arthritis. A randomized controlled trial. JAMA 1999;282:1921-8.

9 Kivitz A, Eisen G, Zhao WW, Bevirt T, Recker DP. Randomized placebocontrolled trial comparing efficacy and safety of valdecoxib with naproxen in patients with osteoarthritis. J Fam Pract 2002;51:530-7.

10 Hudson N, Taha AS, Russell RI, Trye P, Cottrell J, Mann SG, et al. Famotidine for healing and maintenance in nonsteroidal anti- inflammatory drugassociated gastroduodenal ulceration. Gastroenterology 1997;112:1817-22.

11 Emery P, Zeidler H, Kvien TK, Guslandi M, Naudin R, Stead H, et al. Celecoxib versus diclofenac in long-term management of rheumatoid arthritis: randomised double-blind comparison. Lancet 1999;354:2106-11.

12 Hawkey C, Laine L, Simon T, Beaulieu A, Maldonado-Cocco J, Acevedo E, et al. Comparison of the effect of rofecoxib (a cyclooxygenase 2 inhibitor), ibuprofen, and placebo on the gastroduodenal mucosa of patients with osteoarthritis: a randomized, double-blind, placebo controlled trial. Arthritis Rheum 2000;43:370-7.

13 Laine L, Harper S, Simon T, Bath R, Johanson J, Schwartz H, et al. A randomized trial comparing the effect of rofecoxib, a cyclooxygenase 2specific inhibitor, with that of ibuprofen on the gastroduodenal mucosa of patients with osteoarthritis. Rofecoxib Osteoarthritis Endoscopy Study Group. Gastroenterology 1999;117:776-83.

14 Hawkey C. NSAIDs and COX-2 inhibitors: what can we learn from large outcomes trials? The gastroenterologist's perspective. Clin Exp Rheumatol 2001; 19:S23-30.

15 Griffin MR. Epidemiology of nonsteroidal anti-inflammatory drug-associated gastrointestinal injury. Am J Med 1998;104:235-95.

16 Hawkey CJ, Langman MJ. Non-steroidal anti-inflammatory drugs: overall risks and management. Complementary roles for COX-2 inhibitors and proton pump inhibitors. Gut 2003;52:600-8.

17 Office of Population Censuses and Surveys. Mortality statistics: cause. Review of the Registrar General on deaths by cause, sex and age in England and Wales, 1993. London: HMSO, 1995.

18 Wallace J. Cooperative modulation of gastrointestinal mucosal defence by prostaglandins and nitric oxide. Clin Invest Med 1996;19:346-51.

19 Lanas A, Bajador E, Serrano P, Fuentes J, Carreno S, Guardia J, et al. Nitrovasodilators, low-dose aspirin, other nonsteroidal antiinflammatory drugs, and the risk of upper gastrointestinal bleeding. N Engl J Med 2000;343:834-9.

20 Fiorucci S, Antonelli E, Burgaud J, Morelli A. Nitric oxide-releasing NSAIDs. A review of their current status. Drug Saf 2001;24:801-11.

21 Berndt G, Grosser N, Hoogstraate J, Schröder H. A common pathway of nitric oxide release from AZD3582 and glyceryl trinitrate. Eur J Pharm Sci 2004;21:331-5.

22 Berge O-G, Raud J, Hoogstraate J, Wallace J. The CINOD AZD3582 inhibits cyclooxygenase and reduces pain, inflammation and fever in the rat. Eur J Pharm Sci 2002;17(suppl):S77

23 Berge O-G, Raud J, Hoogstraate J. AZZD3582 reduces pain, inflammation and fever in animals. Ann Rheum Dis 2002;61(suppl 1):286.

24 Davies N, Roseth A, Appleyard C, McKnight W, Del Soldato P, Calignano A, et al. NO-naproxen vs. naproxen: ulcerogenic, analgesic and antiinflammatory effects, Aliment Pharmacol Ther 1997; 1:69-79.

25 Muscara MN, McKnight W, Del Soldato P, Wallace JL. Effect of a nitric oxidereleasing naproxen derivative on hypertension and gastric damage induced by chronic nitric oxide inhibition in the rat. Life Sci 1998;62:PL235-40.

26 Öjteg G, Hällgren A, Hoogstraate J. AZD3582, a COX-inhibiting nitric oxide donator (CINOD), is gastroprotective in the rat. Ann Rhuem Dis 2002;61(suppl 1):288. 
27 Hawkey C, Jones JI, Atherton CT, Skelly MM, Bebb JR, Fagerholm U, et al. Gastrointestinal safety of AZD3582: a cyclooxygenase inhibiting nitric oxid donator: proof of concept in humans. Gut 2003;52:1537-42.

28 Lanza FL, Rack MF, Simon TJ, Quan H, Bolognese JA, Hoover ME, et al. Specific inhibition of cyclooxygenase- 2 with MK-0966 is associated with less gastroduodenal damage than either aspirin or ibuprofen. Aliment Pharmacol Ther 1999; 13:761-7.

29 Svedlund J, Sjodin I, Dotevall G. GSRS-a clinical rating scale for gastrointestinal symptoms in patients with irritable bowel syndrome and peptic ulcer disease. Dig Dis Sci 1988;33:129-34.

30 Bellamy N, Buchanan WW, Goldsmith CH, Campbell J, Stitt LW. Validation study of WOMAC: a health status instrument for measuring clinically important patient relevant outcomes to antirheumatic drug therapy in patients with osteoarthritis of the hip or knee. J Rheumatol 1988;15:1833-40.

31 Ware JE, Sherbourne CD. The MOS 36-item short-form health survey (SF-36) I. Conceptual framework and item selection. Med Care 1992;30:473-83.

32 Berndt G, Grosser N, Hoogstraate J, Schröder J. A common pathway for nitric oxide release from AZD3582 and glyceryl trinitrate. Ann Rheum Dis 2002:61(suppl 1):269.

33 Hunt RH, Harper S, Callegari P, Yu C, Quan H, Evans J, et al. Complementary studies of the gastrointestinal safety of the cyclo-oxygenase-2-selective inhibitor etoricoxib. Aliment Pharmacol Ther 2003;17:201-10.

34 Goldstein JL, Correa P, Zhao WW, Burr AM, Hubbard RC, Verburg KM, et al. Reduced incidence of gastroduodenal ulcers with celecoxib, a novel cyclooxygenase-2 inhibitor, compared to naproxen in patients with arthritis. Am J Gastroenterol 2001;96:1019-27.

35 Vioxx. Rofecoxib tablets and oral suspension. Prescribing information. Whitehouse Station, New Jersey: Merck and Co, 1999.

36 Hawkey CJ, Laine L, Harper SE, Quan HUI, Bolognese JA, Mortensen E, for the Rofecoxib Osteoarthritis Endoscopy Multinational Study Group. Influence of risk factors on endoscopic and clinical ulcers in patients taking rofecoxib or ibuprofen in two randomized controlled trials. Aliment Pharmacol Ther 2001;15:1593-601

37 Lanza FL, Graham DY, Davis RE, Rack MF. Endoscopic comparison of cimetidine and sucralfate for prevention of naproxen-induced acute gastroduodenal injury. Effect of scoring method. Dig Dis Sci 1990;35:1494-9.

38 Biarnason I, Macpherson A, Rotman H, Schupp J, Hayllar J. A randomized double-blind, crossover comparative endoscopy study on the gastroduodenal tolerability of a highly specific cyclooxygenase-2 inhibitor, flosulide, and naproxen. Scand J Gastroenterol 1997;32:126-30.

39 Talley NJ, Fullerton S, Junghard O, Wiklund I. Quality of life in patients with endoscopy-negative heartburn: reliability and sensitivity of disease-specific instruments. Am J Gastroenterol 2001;96:1998-2004.

40 Talley NJ, Meineche-Schmidt V, Pare P, Duckworth M, Raisanen P, Pap A, et al. Efficacy of omeprazole in functional dyspepsia: double-blind, randomized, placebo-controlled trials (the Bond and Opera studies). Aliment Pharmacol Ther 1998;12:1055-65. 